PROCEEDINGS OF THE

AMERICAN MATHEMATICAL SOCIETY

Volume 125, Number 3, March 1997, Pages 669-677

S 0002-9939(97)03790-8

\title{
ON ISAACS' THREE CHARACTER DEGREES THEOREM
}

\author{
YAKOV BERKOVICH
}

(Communicated by Ronald M. Solomon)

\begin{abstract}
Isaacs has proved that a finite group $G$ is solvable whenever there are at most three characters of pairwise distinct degrees in $\operatorname{Irr}(G)$ (Isaacs' three character degrees theorem). In this note, using Isaacs' result and the classification of the finite simple groups, we prove the solvability of $G$ whenever $\operatorname{Irr}(G)$ contains at most three monolithic characters of pairwise distinct degrees. $\S 2$ contains some additional results about monolithic characters.
\end{abstract}

$\S 1$.

Let $\operatorname{Irr}(G)$ be the set of all irreducible characters of a finite group $G$, and let $\operatorname{cd}(G)=\{\chi(1) \mid \chi \in \operatorname{Irr}(G)\}$. Isaacs' theorem claims that $G$ is solvable whenever $|\operatorname{cd}(G)| \leq 3$.

A group $G$ is said to be a monolith if it contains only one minimal normal subgroup. It is convenient to consider the group $\{1\}$ as a monolith. A character $\chi$ of $G$ is said to be monolithic if $\chi \in \operatorname{Irr}(G)$ and $G / \operatorname{ker}(\chi)$ is a monolith. Obviously, a monolith possesses a faithful irreducible character (of course, this character is monolithic). Let

$$
\operatorname{Irr}_{m}(G)=\{\chi \in \operatorname{Irr}(G) \mid \chi \text { is monolithic }\}, \operatorname{cd}_{m}(G)=\left\{\chi(1) \mid \chi \in \operatorname{Irr}_{m}(G)\right\} .
$$

Let $p$ denote a prime number. Denote by $G\left(p^{\prime}\right)$ the intersection of kernels of all the nonlinear irreducible characters $\chi$ of $G$ such that $p \nmid \chi(1)$. It is easy to see that $p \mid \phi(1)$ for all nonlinear $\phi \in \operatorname{Irr}\left(P G\left(p^{\prime}\right)\right)$, where $P \in \operatorname{Syl}_{p}(G)$. Therefore $G\left(p^{\prime}\right)$ is $p$-nilpotent and solvable (see [Isa], Corollary 12.2, and [Ber2], Proposition 9 and the Remark, following it). Let $\Phi(G), \mathrm{F}(G)$ be the Frattini subgroup and the Fitting subgroup of $G$, respectively. It follows from the properties of the Frattini subgroup that $G$ is $p$-nilpotent if and only if $G / \Phi(G)$ is p-nilpotent.

Note that all irreducible characters of $p$-groups are monolithic. $\left|\operatorname{cd}_{m}(G)\right|=1$ if and only if $G$ is abelian. Note that $\bigcap_{\chi \in \operatorname{Irr}_{m}(G)} \operatorname{ker} \chi=\{1\}$ (Lemma 2(a)), so that $G$ is a subgroup of a direct product of monoliths. Therefore the set $\operatorname{Irr}_{m}(G)$ is sufficiently large to have a strong influence on the structure of $G$. On the other hand, the following examples show that in many cases the set $\operatorname{cd}_{m}(G)$ is very small compared with $\operatorname{cd}(G)$.

Received by the editors September 5, 1995.

1991 Mathematics Subject Classification. Primary 20C15.

Key words and phrases. Monolith, monolithic character, automorphism group, classification of finite simple groups.

The author was supported in part by the Ministry of Absorption of Israel.

(C)1997 American Mathematical Society 
Examples. 1. Let $G=\mathrm{SL}(2,5) \times \mathrm{L}_{2}(7)$. Then $|\operatorname{Irr}(G)|=54,\left|\operatorname{Irr}_{m}(G)\right|=14$.

2. Let $p_{1}, \ldots, p_{n}$ be pairwise distinct odd primes, $G_{i}$ a dihedral group of order $2 p_{i}(i=1, \ldots, n)$, and $G=G_{1} \times \cdots \times G_{n}$. Then $|\operatorname{cd}(G)|=n+1,\left|\operatorname{cd}_{m}(G)\right|=2$.

3. Let $p_{1}, \ldots, p_{n}$ be pairwise distinct odd primes, $4 \mid p_{i}$ for $i>1$. Let $G_{1}$ be a dihedral group of order $2 p_{1}$, and $G_{i}$ be a Frobenius group of order $4 p_{i} \quad(i=$ $2, \ldots, n)$. Put $G=G_{1} \times \cdots \times G_{n}$. Then $|\operatorname{cd}(G)|=2 n,\left|\operatorname{cd}_{m}(G)\right|=3$.

These examples show that in many cases $\operatorname{Irr}_{m}(G)$ is a rather small subset of $\operatorname{Irr}(G)$.

As usual we consider $\operatorname{Irr}(G / N)$ as a subset of $\operatorname{Irr}(G)$.

This paper was inspired by Chapter 12 of Isaacs' book [Isa] and some old results of Isaacs and Passman.

We collected some known results in the following

Lemma 1. Let $N$ be a normal subgroup of $G$.

(a) (Gallagher; [Isa], Corollary 6.17) Let $\chi \in \operatorname{Irr}(G)$. If $\chi_{N} \in \operatorname{Irr}(N)$, then $\chi \vartheta \in \operatorname{Irr}(G)$ for all $\vartheta \in \operatorname{Irr}(G / N)$.

(b) ([Ber1], Remark 1) If $N$ is nonsolvable, then

$$
|\{\chi(1) \mid \chi \in \operatorname{Irr}(G)-\operatorname{Irr}(G / N)\}|>1 .
$$

(c) ([BZ1], Proposition 30.18) Let $p$ be a prime. If $p \mid \chi(1)$ for every nonlinear $\chi \in \operatorname{Irr}_{m}(G)$, then $G$ is p-nilpotent and solvable.

(d) ([BZ1], Proposition 30.18) $p \nmid \chi(1)$ for every $\chi \in \operatorname{Irr}_{m}(G)$ if and only if a Sylow p-subgroup is normal in $G$ and abelian.

(e) (Broline-Garrison, see [Isa], Theorem 12.19) Let $\chi \in \operatorname{Irr}(G)$ and $K=\operatorname{ker}(\chi)$. Either of the following conditions guarantees the existence of $\psi \in \operatorname{Irr}(G)$ with $\psi(1)>$ $\chi(1)$ and $\operatorname{ker}(\psi)<K$ :

(1e) $K \not \leq \mathrm{F}(G)$.

(2e) $K=\mathrm{F}(G), G / K>\{1\}$ is solvable.

Proof. We prove (c) only. Let $G$ be a counterexample of minimal order. Then $G$ is a monolith. Let $R$ be a minimal normal subgroup of $G$. Since $Y=\operatorname{Irr}(G)-$ $\operatorname{Irr}(G / R) \subseteq \operatorname{Irr}_{m}(G)$, it follows that $p \mid \chi(1)$ for all $\chi \in Y$. Hence, $R \leq G\left(p^{\prime}\right)$ so that $R$ is solvable. Since by induction $G / R$ is solvable and $p$-nilpotent and $G$ is a counterexample, $R$ is a $p$-subgroup. Since $G$ is not $p$-nilpotent, $R \not \leq \Phi(G)$. Take $P \in \operatorname{Syl}_{p}(G)$. It follows from the modular law that $R \not \leq \Phi(P)$. In particular, $R \not P^{\prime}$. Take a linear character $\lambda$ of $P$ such that $R \not \operatorname{ker}(\lambda)$. Since $p \nmid \lambda^{G}(1)$, there exists $\chi \in \operatorname{Irr}\left(\lambda^{G}\right)$ such that $p \nmid \chi(1)$. By reciprocity $R \not \leq \operatorname{ker}(\chi)$, so that $\chi$ is monolithic. Since $R \leq G^{\prime}$, it follows that $G^{\prime} \not \leq \operatorname{ker}(\chi)$, and so $\chi \in \operatorname{Irr}_{m}(G)$ is nonlinear - a contradiction.

Lemma 1(c) generalizes Thompson's Theorem (see [Isa], Corollary 12.2). Lemma $1(\mathrm{~d})$ is a generalization of Michler's result [Mic].

Our principal aim is to prove the following.

Theorem. If $\left|\operatorname{cd}_{m}(G)\right| \leq 3$, then $G$ is solvable.

Proof. Let $G$ be a counterexample of minimal order. Since $\left|\operatorname{cd}_{m}(G / N)\right| \leq\left|\operatorname{cd}_{m}(G)\right|$, it follows from the induction hypothesis that $G$ is a monolith. Let $R$ be a minimal normal subgroup of $G$. Then $G / R$ is solvable and $R=R_{1} \times \cdots \times R_{s}$, where $R_{1}, \ldots, R_{s}$ are isomorphic nonabelian simple groups. Obviously, $R=R^{\prime} \leq G^{\prime}$. 
If $R=G^{\prime}$, then every nonlinear irreducible character of $G$ is monolithic. Therefore $|\operatorname{cd}(G)|=\left|\operatorname{cd}_{m}(G)\right| \leq 3$, and $G$ is solvable by Isaacs' three character degrees theorem. Thus,

(i) $R<G^{\prime}$.

In particular, $G / R$ is nonabelian. Let $H / R$ be a normal subgroup of $G / R$ such that $G / H$ is nonabelian, but every proper epimorphic image of $G / H$ is abelian. By [Isa], Lemma $12.3, G / H$ is a $p$-group, or $G / H=(B / H, L / H)$ is a Frobenius group with cyclic complement $B / H$ and kernel $L / H=(G / H)^{\prime}$; obviously, $G / H$ is a monolith. In what follows we fix so-defined subgroups $H, B$ and $L$.

By [Isa], Lemma 12.3, $\operatorname{cd}(G / H)=\{1, t\}, t>1$.

Let $\phi$ be a nonlinear irreducible character of $G / H$. Then $\phi$ is monolithic, so that $\phi(1)=t \in \operatorname{cd}_{m}(G)$. Obviously, $1 \in \operatorname{cd}_{m}(G)$. By Lemma 1(b), there exist $\chi, \tau \in \operatorname{Irr}(G)-\operatorname{Irr}(G / R)$ such that $\chi(1) \neq \tau(1)$. Obviously, $\chi, \tau \in \operatorname{Irr}_{m}(G)$ and $\chi, \tau$ are nonlinear. Thus, $\operatorname{cd}_{m}(G)=\{1, \chi(1), \tau(1)\}$. We assume that $\chi(1)=t$. Put $\tau(1)=h$. Thus,

(ii) $\operatorname{cd}_{m}(G)=\{1, h, t\}, 1 \neq h \neq t \neq 1$.

We fix so chosen $\phi, \chi, \tau$.

By Lemma 1(c),

(iii) $(h, t)=1$.

Suppose that $G / H$ is a $p$-group. Then $t=p^{\alpha}, p \nmid h$, and $\tau_{H} \in \operatorname{Irr}(H)$ by Clifford theory. Therefore $\tau \phi \in \operatorname{Irr}(G)$ by Lemma 1(a) (recall that $\phi$ is a nonlinear irreducible character of $G / H)$. Since $R \not \leq \operatorname{ker}(\phi \tau)$, it follows that $\phi \tau \in \operatorname{Irr}_{m}(G)$, and so $p^{\alpha} h \in \operatorname{cd}_{m}(G)=\left\{1, p^{\alpha}, h\right\}-$ a contradiction. Therefore,

(iv) $G / H=(B / H, L / H)$ is a Frobenius group, $L / H=(G / H)^{\prime}$ is the unique minimal normal subgroup of $G / H, B / H$ is cyclic of order $t$, and $L / H$ is a $p$-group, where $p$ is a prime number.

Let a nonprincipal $\psi \in \operatorname{Irr}(R), \vartheta \in \operatorname{Irr}\left(\psi^{G}\right)$. Since $R \not \leq \operatorname{ker}(\vartheta)$, it follows that $\vartheta$ is monolithic and $\vartheta(1) \in\{t, h\}$. By Clifford's theorem $\psi(1) \mid t$ or $\psi(1) \mid h$. By (iii), $\operatorname{cd}(R)=M \cup N$ is a nontrivial partition such that inclusions $a \in M, b \in N$ imply $(a, b)=1$. It follows from the description of irreducible characters of direct products that

(v) $s=1$, i.e., $R$ is a simple group.

Since $\chi_{R}$ is reducible by Lemma 1(a), we have, by Clifford's theorem,

(vi) There is a nonprincipal character $\lambda$ in $\operatorname{Irr}(R)$ such that $\lambda(1) \mid t$ and $\lambda(1)<t$. In particular, $t>3$.

If $\lambda$ is as in (vi), then $\lambda(1)>2$ (Klein's theorem; see [Isa], Theorem 14.23), hence we obtain the following stronger inequality:

(vii) $t \geq 6$.

Since $G$ is a monolith, $\mathrm{C}_{G}(R)=\{1\}$, where $\mathrm{C}_{G}(R)$ is the centralizer of $R$ in $G$. Therefore, $G / R$ is a nonabelian subgroup of $A=\operatorname{Out}(R)=\operatorname{Aut}(R) / R$. In particular, $A$ is nonabelian.

In what follows, we use some information about outer automorphism groups of simple groups (see [Gor], §4.15A, and [LPS], Table 2.1).

Let $X$ be a simple group of Lie type defined over a Galois field $\operatorname{GF}\left(p^{e}\right)$. Then $A=\operatorname{Out}(X)=\operatorname{Aut}(X) / X$ is a solvable group with normal subgroups $D$ and $D F$, where $D$, the group of diagonal automorphisms, is abelian of order $d$, and $F$, the group of field automorphisms, is cyclic of order $e$. Also, $A / D F \cong\{1\}, \mathrm{C}(2), \mathrm{S}_{3}$, where $\mathrm{C}(2)$ is cyclic of order 2 , and $\mathrm{S}_{3}$ is the symmetric group of degree 3 . Next, 
$A / D$ is abelian, unless $A / D F \cong \mathrm{S}_{3}$. If $A / D F \cong \mathrm{S}_{3}$, then $A / D$ contains an abelian subgroup of index 2.

If $X$ is sporadic or alternating, then $A$ is abelian. By (i), $R$ is neither sporadic nor alternating.

Putting $q=p^{e}$ we have to consider as $R$ the following groups (see [LPS], Table 2.1):

$$
\begin{gathered}
\mathrm{L}_{n}(q), n \geq 3 ; \mathrm{U}_{n}(q), n \geq 3 ; \mathrm{P} \Omega_{2 m}^{ \pm}(q), m \geq 4 ; \\
\mathrm{E}_{6}(q), 3\left|q-1 ;{ }^{2} \mathrm{E}_{6}(q), 3\right| q+1 .
\end{gathered}
$$

It follows from (iv) that $t|| L / H \mid-1$. By (vii), $t \geq 6$. Therefore by [LPS], Table 2.1 , we have $R \cong \mathrm{L}_{n}(q), n \geq 3$, or $R \cong \mathrm{U}_{n}(q), n \geq 3$. Then $|A|=2 e d,|D|=d$ (where $A=\operatorname{Out}(R)$ ). In the first case $d=(n, q-1)$, in the second one $d=(n, q+1)$. In particular, $d \leq n$. If $D F$ is abelian (we use for $G$ the same notation as before for $X$ ), then $t=2$ by Ito's theorem on degrees of irreducible characters (see [Isa], Theorem 6.15), contradicting (vii). Thus, $D F$ is nonabelian. In particular (recall that $F$ is cyclic), $D \not Z \mathrm{Z}(D F), d>2$. Now, $G / H=T$ is an epimorphic image of a subgroup of $A$, and $T$ is a Frobenius group with kernel $T^{\prime}$ of prime order $r \mid d$ by (iv) (recall that in the case under consideration $D$ is cyclic and $A^{\prime} \leq D$ ). Then $\operatorname{cd}_{m}(T)=\{1, t\}, t \mid r-1$. Since $r \leq d \leq n$, it follows that $t \leq n-1$. Therefore, by (vii), $2<\lambda(1) \leq \frac{n-1}{2}$ and $n \geq 7$ (recall that a nonprincipal $\lambda \in \operatorname{Irr}\left(\chi_{R}\right)$ ). Obviously, $R$ contains a subgroup $S \cong \mathrm{A}_{n}$ (recall that a permutation matrix is unitary). Since $R$ is simple, $S \not \leq \operatorname{ker}(\lambda)$, hence $\operatorname{Irr}\left(\lambda_{S}\right)$ contains a nonlinear character $\mu$. Since $\mu(1) \leq \lambda(1) \leq \frac{n-1}{2}$, it follows that $\operatorname{Irr}\left(\mathrm{A}_{n}\right)$ contains a nonlinear irreducible character of degree at most $\frac{n-1}{2}$, but this is impossible (we use the representation theory of the symmetric groups). The proof is completed.

Conjecture 1. Let $N$ be a normal subgroup of $G$. If

$$
\mathrm{c}(G, N)=|\{\chi(1) \mid \chi \in \operatorname{Irr}(G)-\operatorname{Irr}(G / N)\}|<3,
$$

then $N$ is solvable.

Of course, Conjecture 1 implies the Theorem. If $N$ is nonsolvable, then Lemma 1(b) implies c $(G, N) \geq 2$.

Considering a minimal counterexample $G$ to Conjecture 1, we may assume that $G$ is a monolith such that its minimal normal subgroup $R=N$. As in the proof of the Theorem, we can prove that $R$ is simple. Further on, $\mathrm{C}_{G}(R)=\{1\}$ so $G / R$ is solvable by the classification of the finite simple groups. We consider this Conjecture as a very difficult one.

Conjecture 2. If $\left|\operatorname{cd}_{m}(G)\right| \leq 3$, then the derived length of $G$ does not exceed 3 (Proposition 4(d) gives a weaker result in our case).

We note that the derived length of $G$ does not exceed 3 if $|\operatorname{cd}(G)| \leq 3$ (Isaacs; see [Isa], Theorem 12.15), but there is yet an unproven conjecture that the inequality $\operatorname{dl}(G) \leq|\operatorname{cd}(G)|$ is true for all solvable groups $G$.

Question 1. Let $N$ be a proper normal subgroup of $G$. Suppose that

$$
\left|\left\{\chi(1) \mid \chi \in \operatorname{Irr}_{m}(G)-\operatorname{Irr}(G / N)\right\}\right|=1 .
$$

Describe the structure of $N$ and $G$ in detail. 
We note that Question 1 is very difficult even if $N$ is a minimal normal subgroup of $G$ (it follows from Lemma 1(b) that $N$ is solvable). Recently the pairs $N \triangleleft G$ were classified such that $|\operatorname{Irr}(G)-\operatorname{Irr}(G / N)|=1$ (Burtzev and Kazarin; first results about the structure of such $G$ were proved by Gagola [Gag]; see also [BCZ], Lemma 2).

Question 2. Prove the Theorem without using the classification of the finite simple groups.

$\S 2$.

In this section we prove some additional results about monolithic characters.

Let $\operatorname{Irr}_{1}(G)$ be the set of all nonlinear irreducible characters of $G$. Put

$$
\begin{gathered}
\operatorname{Irr}_{1, m}(G)=\operatorname{Irr}_{m}(G) \cap \operatorname{Irr}_{1}(G), \\
\mathfrak{D}_{m}(G)=\bigcap_{\chi \in \operatorname{Irr}_{m}(G)} \operatorname{ker}(\chi), \mathfrak{D}_{1, m}(G)=\bigcap_{\chi \in \operatorname{Irr}_{1, m}(G)} \operatorname{ker}(\chi) .
\end{gathered}
$$

Since $G^{\prime}$ is the intersection of the kernels of the linear monolithic characters of $G, \mathfrak{D}_{m}(G)=\mathfrak{D}_{1, m}(G) \cap G^{\prime}$.

Lemma 2. (a) $\mathfrak{D}_{m}(G)=\{1\}$.

(b) $\mathfrak{D}_{1, m}(G) \leq \mathrm{Z}(G)$.

Proof. We may assume that $G>\{1\}$. Let $R$ be a minimal normal subgroup of $G$. Take in $G$ a maximal normal subgroup $L$ such that $R \cap L=\{1\}$. Then $G / L$ is a monolith with minimal normal subgroup $R L / L$. Let $\chi$ be a faithful irreducible character of $G / L$. Since $R$ is arbitrary and $R \not \leq \operatorname{ker}(\chi)$, it follows that $\mathfrak{D}_{m}(G)=\{1\}$, and this proves (a). Furthermore, $\mathfrak{D}_{1, m}(G) \cap G^{\prime}=\mathfrak{D}_{m}(G)=\{1\}$ by (a). Take $d \in \mathfrak{D}_{1, m}(G)$ and $x \in G$. Then $[x, d] \in \mathfrak{D}_{1, m}(G) \cap G^{\prime}=\{1\}$, and this proves (b).

The group $G=\mathrm{SL}(2,5) \times \mathrm{C}(3)$, where $\mathrm{C}(3)$ is a cyclic group of order 3 , shows that the inclusion $\mathfrak{D}_{1, m}(G)<\mathrm{Z}(G)$ is possible.

Proposition 3. If $G$ is nonabelian and $\left|\operatorname{Irr}_{1, m}(G)\right|=1$, then one of the following assertions holds:

(a) $G=\operatorname{ES}(m, 2) \times A$, where $\operatorname{ES}(m, 2)$ is an extraspecial group of order $2^{1+2 m}$ and $A$ is abelian of odd order.

(b) $G / \mathrm{Z}(G) \cong \mathrm{AGL}\left(1, p^{\alpha}\right), G^{\prime} \cap \mathrm{Z}(G)=\{1\}, G^{\prime} \cong \mathrm{E}\left(p^{\alpha}\right)$, the elementary group of order $p^{\alpha}, G=G_{1} \times P$, where $P \in \operatorname{Syl}_{p}(\mathrm{Z}(G))$.

Proof. Put $\operatorname{Irr}_{1, m}(G)=\{\chi\}$. Then $\operatorname{ker}(\chi) \leq \mathrm{Z}(G)$ (Lemma 2(b)) and $G / \operatorname{ker}(\chi)$ is a monolith. Let $H / \operatorname{ker}(\chi)$ be a normal subgroup of $G / \operatorname{ker}(\chi)$ such that $G / H$ is nonabelian, but every proper epimorphic image of $G / H$ is abelian. Then $G / H$ is a monolith, so there exists $\tau \in \operatorname{Irr}_{1, m}(G / H)$. By assumption, $\chi=\tau$, hence $H=\operatorname{ker}(\chi)$.

Assume that $G / H$ is nilpotent. As a monolith, $G / H$ is primary. Since $\operatorname{Irr}_{1, m}(G / H)=\operatorname{Irr}_{1}(G / H)$, we have $G / H \cong \mathrm{ES}(m, 2)$ by [Sei]. Since $H \leq \mathrm{Z}(G)$ (Lemma 2(b)), $G$ is nilpotent. In this case $G=P \times A$, where $P \in \operatorname{Syl}_{2}(G)$, $A$ is abelian. Since $\left|\operatorname{Irr}_{1, m}(P)\right|=\left|\operatorname{Irr}_{1, m}(G / A)\right| \leq\left|\operatorname{Irr}_{1, m}(G)\right|=1$, we have $P \cong \operatorname{ES}(m, 2)$, and $G$ is a group from (a). 
Assume that $G / H$ is nonnilpotent. Then by [Isa], Theorem 12.3 , and [Sei], $G / H \cong \operatorname{AGL}\left(1, p^{\alpha}\right)$. By Lemma $2(\mathrm{~b}), H=\mathrm{Z}(G)=Q \times P$, where $P \in \operatorname{Syl}_{p}(\mathrm{Z}(G))$ (in fact, $\mathrm{Z}(G / H)=\{1\}$ implies $\mathrm{Z}(G) \leq H)$. Suppose that $Q=\{1\}$. Then $P=$ $\mathfrak{D}_{1, m}(G)$ and $P \cap G^{\prime}=\{1\}$ (see the proof of Lemma 2). Then $G^{\prime} \cong \mathrm{E}\left(p^{\alpha}\right)$ and $G^{\prime} P=G^{\prime} \times P$ is abelian. By [Hup], Satz 1.17.4(a), $G=G_{1} \times P$. Analogously we consider the case $Q>\{1\}$.

Let $G=G_{1} \times P, G_{1} / \mathrm{Z}\left(G_{1}\right) \cong \operatorname{AGL}\left(1, p^{\alpha}\right), P \in \operatorname{Syl}_{p}(\mathrm{Z}(G))$. It is easy to check that $\left|\operatorname{Irr}_{1, m}(G)\right|=1$.

Question 3. Classify the groups $G$ such that $\left|\operatorname{Irr}_{1, m}(G)\right| \leq 3$.

Question 4. Classify the groups $G$ such that any two distinct characters in $\operatorname{Irr}_{1, m}(G)$ have distinct degrees.

If any two distinct characters in $\operatorname{Irr}_{1}(G)$ have distinct degrees, then

$$
G \in\left\{\operatorname{ES}(m, 2), \operatorname{AGL}\left(1, p^{\alpha}\right),(\mathrm{Q}(8), \mathrm{E}(9))\right\},
$$

where $\mathrm{Q}(8)$ is the ordinary quaternion group, $\mathrm{E}(9)$ is elementary of order 9 , and $(A, B)$ is a Frobenius group with kernel $B$ and complement $A[\mathrm{BCH}]$.

Let $\operatorname{dl}(G)$ and $\operatorname{nl}(G)$ denote the derived length and the nilpotent length of a solvable group $G$, respectively.

Proposition 4. (a) If $\left|\operatorname{Irr}_{1, m}(G)\right| \leq 4$, then $G$ is solvable, unless $G=G^{\prime} \times A$, where $G^{\prime} \cong \mathrm{L}_{2}(5)$.

(b) If $\left|\operatorname{cd}_{m}(G)\right|=2$, then $\operatorname{dl}(G)=2$.

(c) If all characters in $\operatorname{Irr}_{1, m}(G)$ have distinct degrees, then $G$ is solvable.

(d) If $G$ is solvable, then $\left|\operatorname{cd}_{m}(G)\right| \geq \operatorname{nl}(G)$.

Proof. Assume that in all cases $G$ is a counterexample of minimal order. Then, in cases (b), (c) and (d), $G$ is a monolith. Let $R$ be a minimal normal subgroup of $G$. Set $|R|=r^{\alpha}$ if $R$ is solvable, where $r$ is a prime.

(a) If $\operatorname{Irr}_{1}(G)=\operatorname{Irr}_{1, m}(G)$, then $G \cong \mathrm{L}_{2}(5)$ by [Ber3] or [Ber4]. Thus, $\operatorname{Irr}_{1, m}(G)$ $\subset \operatorname{Irr}_{1}(G)$.

(a.i) Suppose that $G$ is a monolith.

It follows from $\operatorname{Irr}_{1, m}(G) \subset \operatorname{Irr}_{1}(G)$ that $R<G^{\prime}$, and so $G / R$ is nonabelian. In particular, $\left|\operatorname{Irr}_{1, m}(G / R)\right| \geq 1$.

Assume that $R$ is nonsolvable. Let $\phi_{1}, \ldots, \phi_{k} \in \operatorname{Irr}_{1}(R)$ be such that $\phi_{1}(1)<$ $\cdots<\phi_{k}(1)$. If $\chi^{i} \in \operatorname{Irr}\left(\phi_{i}^{G}\right)$ for all $i$, then $\chi^{1}, \ldots, \chi^{k}$ are distinct nonlinear monolithic characters of $G$ by reciprocity. By Isaacs' three character degree theorem (or by the Theorem), $k \geq 3$. It follows from $\left|\operatorname{Irr}_{1, m}(G)\right| \leq 4$ that $\left|\operatorname{Irr}_{1, m}(G / R)\right|=1$, and so $k=3$. Therefore, $R$ is simple, and, by Proposition $3, G / R$ is solvable. Let $H / R$ be a maximal normal subgroup of $G / R$. Then $|G: H|=p$, a prime number. If, say, $\left(\chi^{1}\right)_{H} \in \operatorname{Irr}(H)$, then $\left(\left(\chi^{1}\right)_{H}\right)^{G}=\chi_{1}^{1}+\cdots+\chi_{p}^{1}$, where $\chi_{j}^{1} \in \operatorname{Irr}_{1, m}(G)$ are distinct of the same degree for all $j$. Since $\chi^{2}, \chi^{3} \notin\left\{\chi_{1}^{1}, \ldots, \chi_{p}^{1}\right\}$, it follows that $\left|\operatorname{Irr}_{1, m}(G)\right| \geq 3+1+p-1>4-$ a contradiction. Analogously, $\left(\chi^{2}\right)_{H},\left(\chi^{3}\right)_{H}$ are reducible. Then $\left(\chi^{i}\right)_{H}=\lambda_{1}^{i}+\cdots+\lambda_{p}^{i}$, where $\lambda_{j}^{i} \in \operatorname{Irr}(H)$ are distinct of the same degree for all $i, j$ (by Clifford theory). Therefore $p \mid \chi^{i}(1)$ for $i=1,2,3$. This means that $R \leq G\left(p^{\prime}\right)$. Since $G\left(p^{\prime}\right)$ is solvable, $R$ is solvable as well - a contradiction.

Thus, $R$ is solvable. Since $G / R$ is nonsolvable, we see that $\left|\operatorname{Irr}_{1, m}(G / R)\right| \geq$ 4 by induction. $G$ as a monolith has a faithful irreducible character; therefore, $\left|\operatorname{Irr}_{1, m}(G)\right| \geq\left|\operatorname{Irr}_{1, m}(G / R)\right|+1 \geq 4+1=5$ - a contradiction. 
(a.ii) Suppose that $G$ is not a monolith. Let $R_{1}$ be a minimal normal subgroup of $G, R_{1} \neq R$.

Suppose that one of the subgroups $R, R_{1}$, say $R$, is nonsolvable. Since $R R_{1} / R_{1} \cong$ $R$ is a minimal normal subgroup of $G / R_{1}$, we have, by induction, $R \cong \mathrm{L}_{2}(5)$. Since $\operatorname{Aut}(R) \cong \mathrm{S}_{5}$ satisfies $\left|\operatorname{Irr}_{1, m}\left(\mathrm{~S}_{5}\right)\right|=5$, it follows that $G=R \times \mathrm{C}_{G}(R)$. Since $\left|\operatorname{Irr}_{1, m}\left(G / \mathrm{C}_{G}(R)\right)\right|=4=\left|\operatorname{Irr}_{1, m}(G)\right|$, we obtain $\mathrm{C}_{G}(R)=\mathrm{Z}(G)$ by Lemma 2(b), and the result follows.

Thus, we may assume that all minimal normal subgroups of $G$ are solvable. Then by induction, $G / \mathrm{S}(G) \cong \mathrm{L}_{2}(5)$, and, as in the previous paragraph, $\mathrm{S}(G)=\mathrm{Z}(G)$ (here we use Lemma 2(b)). Obviously, $G^{\prime \prime}=G^{\prime}$. Since, by the above, $G^{\prime}$ is not a minimal normal subgroup of $G$, it is a representation group of $\mathrm{L}_{2}(5)$, i.e., by a known result of I. Schur, $G^{\prime} \cong \operatorname{SL}(2,5)$. We have $G=G^{\prime} \mathrm{Z}(G)$. Instead of $G$ we may consider its epimorphic image $\bar{G}$ such that $\mathrm{Z}(G)$ is a nonidentity cyclic 2-group. Without loss of generality we may assume that $\mathrm{Z}(G)$ is a cyclic 2 -subgroup. It is easy to check that $\left|\operatorname{Irr}_{1, m}(G)\right| \geq\left|\operatorname{Irr}_{1, m}(\mathrm{SL}(2,5))\right|=8$ - a contradiction. The proof of (a) is complete.

(b) The group $G$ is solvable by the Theorem. If $G$ is nilpotent, it is an $r$-group (recall that $R$ is an $r$-group), $\operatorname{Irr}_{m}(G)=\operatorname{Irr}(G)$, so that $\operatorname{dl}(G)=2$ by Taketa's Theorem (see [Isa], Theorem 5.12). Thus, $G$ is not nilpotent. Let $\operatorname{cd}_{m}(G)=$ $\{1, t\}$. Since $G$ is a monolith, it is not $r$-nilpotent so, by Lemma $1(\mathrm{c}), r \nmid t$. By Lemma 1(d), $T \in \operatorname{Syl}_{r}(G)$ is normal in $G$ and abelian. Since $G$ is a monolith, $T=\mathrm{F}(G)$. Since $R \leq G^{\prime}, G^{\prime} / R$ is abelian by induction, and $G^{\prime}$ is nonabelian by the induction hypothesis, $G^{\prime} \not \leq T$ (in particular, $G^{\prime}$ is nonnilpotent); it follows from the properties of the Frattini subgroup that $R \nless \leq \Phi(G)$. Then there exists a maximal subgroup $M$ of $G$ such that $G=M R, M \cap R=\{1\}$. If $R<T$, then $\mathrm{N}_{G}(M \cap T)=G$ and $M \cap T>\{1\}$, and so $G$ is not a monolith. Thus, $R=T=\mathrm{F}(G)$. Let $H / R$ be a normal subgroup of $G / R$ such that $G / H$ is nonabelian, but every proper epimorphic image of $G / H$ is abelian. Then $G / H$ is a monolith so that $\operatorname{cd}_{m}(G / H)=\operatorname{cd}_{m}(G)=\{1, t\}$. There exists $\chi_{1} \in \operatorname{Irr}(G)$ such that $\operatorname{ker}\left(\chi_{1}\right)=H$ (in particular $\chi_{1} \in \operatorname{Irr}_{1, m}(G)$ ). By Lemma 1(e) there exists $\chi_{2} \in \operatorname{Irr}(G)$ such that $\chi_{2}(1)>\chi_{1}(1)=t$ and $\operatorname{ker}\left(\chi_{2}\right)<H$. By assumption $\chi_{2}$ is not monolithic so that $R<\operatorname{ker}\left(\chi_{2}\right)$. Since $R=\mathrm{F}(G)$, there exists (by Lemma $\left.1(\mathrm{e})\right) \chi_{3} \in \operatorname{Irr}(G)$ such that $\chi_{3}(1)>\chi_{2}(1)>t$ and $\operatorname{ker}\left(\chi_{3}\right)<\operatorname{ker}\left(\chi_{2}\right)$. By asumption, $\chi_{3}$ is not monolithic, so that $R<\operatorname{ker}\left(\chi_{3}\right)$. Continuing in this way we obtain an infinite sequence of nonmonolithic irreducible characters $\left\{\chi_{i}\right\}(i=1,2, \ldots)$ of $G / R$ such that $\chi_{1}(1)<\chi_{2}(2)<\ldots-$ a contradiction.

(c) By induction, $G / R$ is solvable. If $G / R$ is abelian, then $\operatorname{Irr}_{1, m}(G)=\operatorname{Irr}_{1}(G)$, and $G$ is solvable by [BCH]. Hence, $G / R$ is nonabelian. Let $H / R$ be a normal subgroup of $G / R$ such that $|G: H|=p$ is a prime number. Let $\chi \in \operatorname{Irr}(G)-$ $\operatorname{Irr}(G / R)$. If $\chi_{H} \in \operatorname{Irr}(H)$, then $\left(\chi_{H}\right)^{G}=\chi_{1}+\cdots+\chi_{p}$, where $\chi_{1}=\chi, \ldots, \chi_{p} \in$ $\operatorname{Irr}_{1}(G)-\operatorname{Irr}(G / R)$ are distinct of the same degree by reciprocity and Clifford theory. Therefore, $\chi_{1}, \ldots, \chi_{p} \in \operatorname{Irr}_{1, m}(G)$ - a contradiction. Then, by Clifford theory, $\chi_{H}=\lambda_{1}+\cdots+\lambda_{p}$, where $\lambda_{1}, \ldots, \lambda_{p} \in \operatorname{Irr}(H)$ are distinct of the same degree. Thus, $p \mid \chi(1)$ for all $\chi \in \operatorname{Irr}(G)-\operatorname{Irr}(G / R)$. Hence, $R \leq G\left(p^{\prime}\right)$, and $R$ is solvable - a contradiction.

(d) Since $\operatorname{nl}(G / \Phi(G))=\operatorname{nl}(G)$, we obtain $R \not \leq \Phi(G)$ (in fact, by induction, $\left.\operatorname{nl}(G / R) \leq\left|\operatorname{cd}_{m}(G / R)\right|<\left|\operatorname{cd}_{m}(G)\right|\right)$. Let $M$ be a maximal subgroup of $G$ such that $R \not \leq M$. If $R<\mathrm{F}(G)$, then $M \cap \mathrm{F}(G)>\{1\}, \mathrm{N}_{G}(M \cap \mathrm{F}(G)) \geq M$ and 
$\mathrm{N}_{R}(M \cap \mathrm{F}(G))>\{1\}$. So $\mathrm{N}_{G}(M \cap \mathrm{F}(G))=G, G$ is not a monolith - a contradiction. Thus, $\mathrm{F}(G)=R$. Then, by Lemma $1(\mathrm{e}), G$ has a faithful irreducible character $\tau$ such that $\tau(1)>\chi(1)$ for all $\chi \in \operatorname{Irr}(G / R)$. Thus, $\left|\operatorname{cd}_{m}(G / R)\right| \leq$ $\left|\operatorname{cd}_{m}(G)\right|-1=n-1$. Therefore, by induction, $\operatorname{nl}(G / R) \leq\left|\operatorname{cd}_{m}(G / R)\right| \leq n-1$. Hence, $\operatorname{nl}(G)=1+\operatorname{nl}(G / R) \leq 1+(n-1)=n$ - a contradiction.

Proposition 4(d) generalizes Garrison's result (see [Isa], Corollary 12.21). Proposition 4(b) generalizes [Isa], Corollary 12.6.

Remark. Let $G$ be a solvable group such that $\operatorname{nl}(G)=|\operatorname{cd}(G)|=n$. Take a normal subgroup $H$ in $G$ such that $\operatorname{nl}(G / H)=n$, but $\operatorname{nl}(\bar{G})<n$ for every proper epimorphic image $\bar{G}$ of $G / H$. It follows from Lemma $1(\mathrm{e})$ that $H<\mathrm{F}(G)$. But if $\operatorname{nl}(G)=\left|\operatorname{cd}_{m}(G)\right|$, then $H$ is not necessarily nilpotent (see Example 2).

For a monolith $G$, let

$$
\mu(G)=\min \{\chi(1) \mid \chi \in \operatorname{Irr}(G), \chi \text { is faithful }\} .
$$

A subset $X$ of $\operatorname{Irr}_{1, m}(G)$ is said to be fundamental if whenever $\chi \in X$, then $X \cap \operatorname{Irr}_{1, m}(G / \operatorname{ker}(\chi))=\{\chi\}$ and $\chi(1)=\mu(G / \operatorname{ker}(\chi))$. By construction, $|X|$ is the number of normal subgroups $N$ of $G$ such that $G / N$ is a nonabelian monolith.

Let $S$ be a set of simple groups. A tower of groups from $S$ is said to be an $S$-group. We consider $\{1\}$ as an $S$-group.

Proposition 5. Let $X$ be a fundamental subset of $\operatorname{Irr}_{1, m}(G)$. If for every $\chi \in X$ there exist $H \leq G$ and $\lambda \in \operatorname{Irr}(H)$ such that $H / \operatorname{ker}(\lambda)$ is an $S$-group and $\chi=\lambda^{G}$, then $G$ is an $S$-group.

Proof. Let $G$ be a counterexample of minimal order. Then $G$ is a monolith. Let $R$ be a minimal normal subgroup of $G$. By induction, $G / R$ is an $S$-group and, by assumption, $R$ is not an $S$-group. Let $\chi \in X \cap \operatorname{Irr}_{1, m}(G)$. By hypothesis, there exist $H \leq G$ and $\lambda \in \operatorname{Irr}(H)$ such that $H / \operatorname{ker}(\lambda)$ is an $S$-group and $\chi=\lambda^{G}$. Since $\chi$ is a faithful character of $G$ of minimal degree and $\left(1_{H}\right)^{G}$ is reducible, $R \leq \operatorname{ker}\left(\left(1_{H}\right)^{G}\right) \leq H$. Since $H / \operatorname{ker}(\lambda)$ is an $S$-group but $R$ is not an $S$-group, it follows that $R \leq \operatorname{ker}(\lambda)$. Then $\{1\}<R \leq \operatorname{ker}\left(\lambda^{G}\right)=\operatorname{ker}(\chi)=\{1\}-$ a contradiction.

Of course, this proof is a small modification of one of two known proofs of Taketa's Theorem. Proposition 5 generalizes [Ber1], Theorem 2.

Conjecture 3. If $G$ is as in Proposition $4(\mathrm{c})$, then $\operatorname{dl}(G) \leq 3 .^{1}$

Note that $\left|\operatorname{cd}_{m}(G)\right|$ is not bounded for such groups $G$.

Conjecture 4. If $\operatorname{cd}_{m}(G)$ is a chain under divisibility, then $G$ has an ordered Sylow tower.

Conjecture 5. If every monolithic character of $G$ is monomial, then $\operatorname{dl}(G) \leq$ $\left|\operatorname{cd}_{m}(G)\right|$.

Conjecture 6. There exists a constant $c$ such that $\left|\operatorname{cd}_{m}(G)\right| \leq c \cdot \operatorname{dl}(G)$.

Conjecture 7. Let $G$ be nonsolvable. If only two characters in $\operatorname{Irr}_{1, m}(G)$ have equal degrees, then $G=L \times H$, where $L \cong \mathrm{L}_{2}(5)$ or $\mathrm{L}_{2}(7), \operatorname{cd}_{m}(L) \cap \operatorname{cd}_{m}(H)=\{1\}$ and all characters in $\operatorname{Irr}_{1, m}(H)$ have distinct degrees.

\footnotetext{
${ }^{1}$ As proved by L. S. Kazarin and the author, $\operatorname{nl}(G) \leq 3$.
} 
If $G$ is nonsolvable and only two characters in $\operatorname{Irr}_{1}(G)$ have equal degrees, then $G \in\left\{\mathrm{L}_{2}(5), \mathrm{L}_{2}(7)\right\}[\mathrm{BK}]$.

For further information on monolithic characters see [BZ1], Chapter 30 and [BZ2].

I am indebted to the referee for corrections, useful comments and suggestions.

\section{REFERENCES}

[Ber1] Y. Berkovich, Generalizations of M-groups, Proc. Amer. Math. Soc. 123, 11 (1995), 3263-3268. CMP 95:16

[Ber2] Y. Berkovich, Finite groups with small sums of some non-linear irreducible characters, J. Algebra 171 (1995), 426-443. MR 96c:20015

[Ber3] Y. Berkovich, Finite groups with few nonlinear irreducible characters, Izv. SeveroKavkazskogo Tzentra Vyschei Shkoly, estestvennye nauki 1 (1987), 8-13 (Russian). MR 88k:20021

[Ber4] Y. Berkovich, Finite groups with few nonlinear irreducible characters, Problems in group theory and homological algebra, Yaroslav. Gos. Univ., Yaroslavl, 1990, pp. 97-107 (Russian). MR 93d:20015

$[\mathrm{BCH}]$ Y. Berkovich, D. Chillag, and M. Herzog, Finite groups in which the degrees of the nonlinear irreducible characters are distinct, Proc. Amer. Math. Soc. 115 (1992), 955959. MR 92j:20006

[BCZ] Y. Berkovich, D. Chillag, and E. Zhmud', Finite groups in which all nonlinear irreducible characters have three values, Houston Math. J. 21 (1) (1995), 17-28. MR 96i:20005

[BK] Y. Berkovich and L. Kazarin, Finite groups in which only two nonlinear irreducible characters have equal degrees, J. of Algebra 184 (1996), 538-560.

[BZ1] Y. Berkovich and E. Zhmud', Characters of Finite Groups, 2, Amer. Math. Soc. (to appear)

[BZ2] Y. Berkovich and E. Zhmud', On monolithic characters, Houston Math. J. 22 (1996), 263-278.

[Gag] S.C. Gagola, Characters vanishing on all but two conjugacy classes, Pacific J. Math 109 (1983), 263-285. MR 85e:20009

[Gor] D. Gorenstein, Finite Simple Groups. An Introduction to Their Classification, Plenum Press, New York, 1982. MR 84j:20002

[Hup] B. Huppert, Endliche Gruppen, Bd. 1, Springer, Berlin, 1967. MR 37:302

[Isa] I.M. Isaacs, Character Theory of Finite Groups, Academic Press, New York, 1976. MR $\mathbf{5 7 : 4 1 7}$

[LPS] M.W. Liebeck, C.E. Praeger, and J. Saxl, The Maximal Factorizations of the Finite Simple Groups and Their Automorphism Groups, Memoirs of the American Mathematical Society, no. 432, Providence, RI, 1990.

[Mic] G.O. Michler, Modular representation theory and the classification of finite simple groups, Proc. Symp. Pure Math. 47 (1987), 223-232. MR 89b:20034

[Sei] G. Seitz, Finite groups having only one irreducible representation of degree greater than one, Proc. Amer. Math. Soc. 19 (1968), 459-461. MR 36:5212 ISRAEL

Department of Mathematics and Computer Science, University of Haifa, Haifa 31905, 